\title{
Assessment of Cases of Cases of Mesenteric Ischaemia with MDCT
}

\author{
Praveen Kumar Monagari ${ }^{1}$ \\ ${ }^{1}$ Associate Professor, Department of Radiology, Surabhi Institute Of Medical Sciences, Siddipet, Medak District.
}

\section{Abstract}

Background: Mesenteric ischaemia comprises a complex, heterogeneous group of disorders that result in inadequate blood supply to the small or large bowel. The present study was conducted to assess the cases of mesenteric ischemia with MDCT. Subjects and Methods: The present study was conducted on 56 cases of mesenteric ischemia of both genders. 128 detector rows Siemens definition AS scanner was used for taking MDCT. Results: Out of 56 patients, males were 32 and females were 24.26 cases were acute and 30 were chronic patients. The difference was non- significant (P- 0.5). MDCT shows bowel distention in 43 cases, bowel wall thickening in 37, SMA dissection in 48, pneumatosis in 13, SMA thrombosis in 17 and pneumoporta in 17 cases. The difference was significant $(\mathrm{P}<0.05)$. Conclusion: Authors found that MDCT is a newer and best diagnostic tool for the detection of mesenteric ischaemia. Bowel changes are well and accurately seen with MDCT.

Keywords: Bowel, Mesenteric ischaemia, MDCT.

Corresponding Author: Dr. Praveen Kumar Monagari, Associate Professor, Department of Radiology, Surabhi Institute Of Medical Sciences, Siddipet, Medak District.

Received: August 2019

Accepted: August 2019

\section{Introduction}

Mesenteric ischaemia comprises a complex, heterogeneous group of disorders that result in inadequate blood supply to the small or large bowel. In the acute setting, mesenteric ischaemia is a surgical emergency, with mortality rates ranging between 30 and $90 \%$. Because the clinical presentation and imaging manifestations of mesenteric ischaemia are variable and often nonspecific, a high index of clinical and radiologic suspicion is required for prompt diagnosis and treatment. ${ }^{[1]}$ The severity of mesenteric ischaemia ranges from localised, transient ischaemia to frank necrosis of the gastrointestinal tract and is a function of multiple factors, including the degree of vascular compromise, duration of the insult, metabolic requirements of the affected bowel and capacity of the underlying systemic circulation, including collateral flow. ${ }^{[2]}$

It is important to notice that the computerized tomography (CT) appearance of acute bowel ischemia will depend on its cause, severity, location and extent. The findings can be heterogeneous and nonspecific. The most frequent cause of acute bowel ischemia is thromboembolism, associated with cardiovascular problems. Other acute conditions are aortic and mesenteric dissection, hypercoagulable state, aortic surgery, decreased cardiac output and bowel obstruction. ${ }^{[3]}$ Multidetector cone beam tomography (MDCT) technology has dramatically improved the performance of CT scan which acquires rapid volumetric data over a large anatomic volume. This allows retrospective multiplanar reformatted images and CT angiograms with 2-D and 3-D visualization. These advantages are helpful in identifying the site, level and cause of bowel ischaemia by showing abnormal segments of bowel, mesentery and vessels. In addition, other pathological conditions can also be detected in patients suspected of mesenteric ischaemia. ${ }^{[4]}$ The present study was conducted to assess the cases of mesenteric ischemia with MDCT.

\section{Subjects and Methods}

The present study was conducted in the department of Radiodiagnosis. It consists of 56 cases of mesenteric ischemia of both genders. All were informed regarding the study and written consent was taken.Ethical clearance was obtainedprior starting the study from institutional ethical committee.

General information such as name, age, gender etc. was recorded. 128 detector rows Siemens definition AS scanner was used for taking MDCT. Examination on CT images was obtained from the dome of the liver to the level of the perineum to cover the entire course of the intestine. With MDCT scanners, a collimation of $0.625 \mathrm{~mm}$ and a detector pitch of 1.0-2.0 were used. Sagittal images were obtained for assessing the origin of the mesenteric arteries and their variation. Results were subjected to statistical analysis. P value less than 0.05 was considered significant. 
Results

Table 1: Distribution of patients

\begin{tabular}{|l|l|l|}
\hline \multicolumn{3}{|c|}{ Total- 56 } \\
\hline Gender & Males & Females \\
\hline Number & 32 & 24 \\
\hline
\end{tabular}

Table 1 shows that out of 56 patients, males were 32 and females were 24 .

\begin{tabular}{|l|l|l|}
\hline Table 2: Type of mesenteric ischemia \\
\hline Acute & Chronic & P value \\
\hline 26 & 30 & 0.12 \\
\hline
\end{tabular}

Table 2 shows that 26 cases were acute and 30 were chronic patients. The difference was non- significant (P- 0.5).

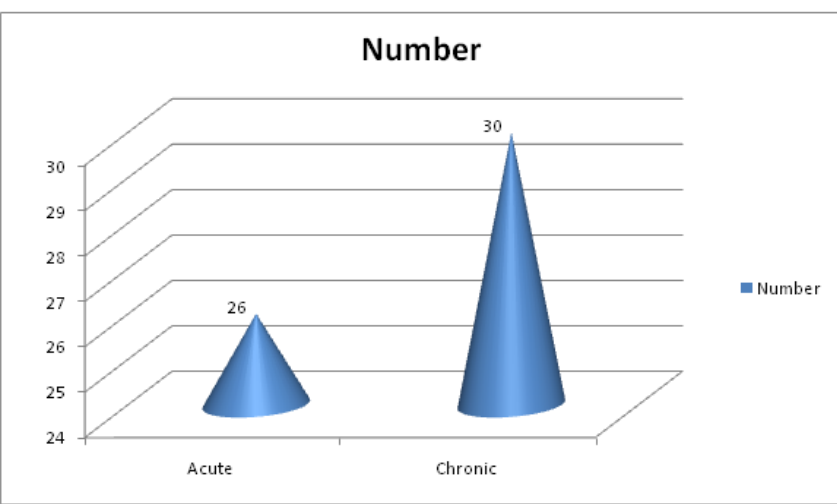

Graph 1: Type of mesenteric ischemia

\begin{tabular}{|c|c|c|}
\hline \multicolumn{3}{|c|}{ Table 3: MDCT findings in mesenteric ischemia } \\
\hline Bowel distension & 43 & \multirow{6}{*}{0.01} \\
\hline Bowel thickening & 37 & \\
\hline SMA dissection & 48 & \\
\hline Pneumatosis & 13 & \\
\hline SMA thrombosis & 17 & \\
\hline Pneumoporta & 17 & \\
\hline
\end{tabular}

Table 1, graph 1 shows that MDCT shows bowel distention in 43 cases, bowel wall thickening in 37, SMA dissection in 48, pneumatosis in 13, SMA thrombosis in 17 and pneumoporta in 17 cases. The difference was significant $(\mathrm{P}<0.05)$.

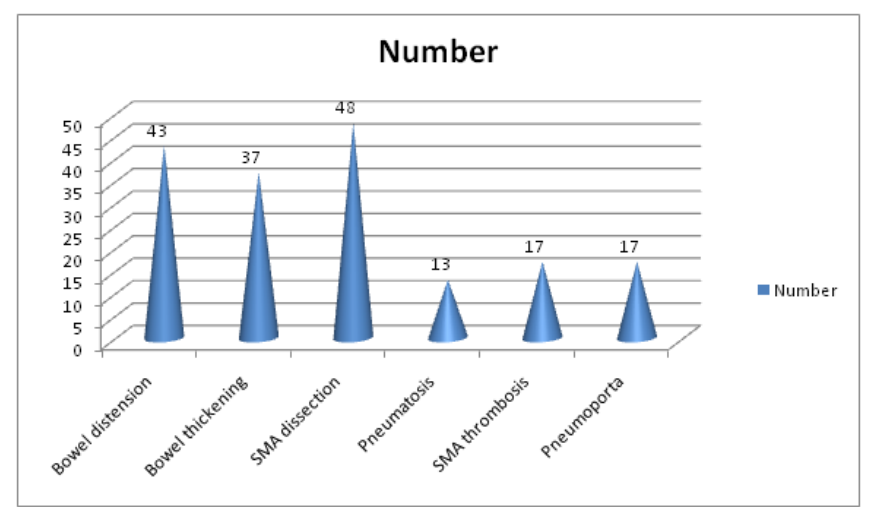

Graph 2: MDCT findings in mesenteric ischemia

\section{Discussion}

Mesenteric ischemia is caused by a reduction in blood flow for intestinal circulation of sufficient magnitude to compromise the viability of the affected portion. The disease can be classified into acute and chronic, based upon the rapidity and the degree to which blood flow is compromised and the duration of the symptoms. Acute and chronic mesenteric ischaemia remains a complex disease entity characterized by perfusion abnormality to the GI tract. Because it presents with nonspecific symptoms and laboratory findings, mesenteric ischaemia remains a clinical diagnostic challenge. ${ }^{[5]}$

There are three stages of acute bowel ischemia. The first stage, which is characterized by mucosal necrosis and mucosal erosion or hemorrhage, is reversible. In stage II, the damage extends to submucosal and muscular layers. Stages I and II are poorly identified on CT. Mucosal enhancement and wall thickening can be detected, but those findings are usually mild and nonspecific. In stage III, which represents transmural bowel wall necrosis, the CT findings are morereliable and specific, and include abnormal or absent wallenhancement, mesenteric stranding, ascites, pneumatosisand portal venous gas. ${ }^{[6]}$ The present study was conducted to assess the cases of mesenteric ischemia with MDCT.

In this study, out of 56 patients, males were 32 and females were 24 . We found that 26 cases were acute and 30 were chronic patients. Mesenteric ischemiacan be caused by thromboembolism, bowel obstruction, abdominal inflammatory disease and trauma. A hyper active stage occurs first, in which the primary symptoms are severe abdominal pain and the passage of bloody stools. Many patients get better and do not progress beyond this phase.A paralytic phase can follow if ischemia continues; in this phase, the abdominal pain becomes more widespread, the belly becomes more tender to the touch, and bowel motility decreases, resulting in abdominal bloating, no further bloody stools, and absent bowel sounds on exam. ${ }^{[7]}$

We found that MDCT shows bowel distention in 43 cases, bowel wall thickening in 37, SMA dissection in 48, pneumatosis in 13, SMA thrombosis in 17 and pneumoporta in 17 cases. Amlendu et $\mathrm{al}^{8}$ retrospectively reviewed 37 cases of mesenteric ischaemia which were diagnosed by MDCT scan during 27 months and were further sent for surgery and or DSA and clinical follow up. These patients were further grouped to acute and chronic depending on stenosis/status of mesenteric vessels, bowel wall changes and collateral pathways, and CT findings were correlated with surgical/DSA findings/followup. Common findings of acute mesenteric ischaemia were bowel wall thickening (80\%), bowel distention $(53.3$ $\%)$ and altered bowel wall enhancement (46.6\%), while other findings SMA dissection, Pneumatosis and 
Pneumoporta were $33.3 \%$. Common findings of chronic mesenteric ischaemia were blocked SMA and collaterals filling post-block SMA $(100 \%)$, stenosis of superior mesenteric artery $(77.3 \%)$, stenosis of IMA $(54.5 \%)$, and stenosis of celiac trunk $(31.8 \%)$.

The diagnosis of CMI is based on clinical symptoms and supported by imaging findings, following exclusion of other potential intestinal disorders. ${ }^{[9]}$ MDCT accurately demonstrates calcified and noncalcified plaque causing arterial stenosis or occlusion, typically in the proximal CA and SMA. Small, attenuated vessels and large collateral vessels are important supportive findings. ${ }^{[10]}$

\section{Conclusion}

Authors found that MDCT is a newer and best diagnostic tool for the detection of mesenteric ischaemia. Bowel changes are well and accurately seen with MDCT.

\section{References}

1. Herbert GS, Steele SR. Acute and chronic mesenteric ischemia. Surg
Clin N Am. 2007;87(5):1115-1134.

2. Gore RM, Yaghmai V, Thakrar KH, Berlin JW, Mehta UK, Newmark GM, et al. Imaging in intestinal ischemic disorders. Radiol Clin N Am. 2008;46(5):845-875.

3. Sreenarasimhaiah J. Chronic mesenteric ischemia. Best Pract Res Clin Gastroenterol. 2005;19(2):283-295.

4. Shih MC, Angle JF, Leung DA, et al. CTA and MRA in mesenteric ischemia: part 2, normal findings and complications after surgical and endovascular treatment. Am J Roentgenol 2007;188(2):462-71.

5. Levy AD. Mesenteric ischemia. Radiol Clin N Am. 2007;45(3):593599.

6. Moschetta M, Telegrafo M, Rella L, et al. Multi-detector CT features of acute intestinal ischemia and their prognostic correlations. World $\mathbf{J}$ Radiol 2011;6(5):130-8.

7. Rha, Johnson PT, Horton KM, et al. Unsuspected mesenteric arterial abnormality: comparison of MDCT axial sections to interactive 3D rendering. Am J Roentgenol 2007;189(4):807-13.

8. Amlendu, Matheson PJ, Franklin GA, Harbrecht BG, Richardson JD, GarrisonRN. Randomized controlled trial evaluating the efficacy of peritonealresuscitation in the management of trauma patients undergoing damage control surgery J Am Coll Surg 2009;224:396404.

9. Cognet F, Ben Salem D, Dranssart M, Cercueil JP, Weiller M, Tatou $\mathrm{E}$, et al. Chronic mesenteric ischemia: imaging and percutaneous treatment. Radiographics. 2002;22(4):863-879/

10. Schieda N, Fasih N, Shabana W. Triphasic CT in the diagnosis of acute mesenteric ischaemia. Eur Radiol. 2013;23(7):1891-1900.

Copyright: ( $)$ the author(s), 2019. It is an open-access article distributed under the terms of the Creative Commons Attribution License (CC BY 4.0), which permits authors to retain ownership of the copyright for their content, and allow anyone to download, reuse, reprint, modify, distribute and/or copy the content as long as the original authors and source are cited.

How to cite this article: Monagari PK. Assessment of Cases of Cases of Mesenteric Ischaemia with MDCT. Asian J. Med. Res. 2019;8(3):RD01-RD03.

DOI: dx.doi.org/10.21276/ajmr.2019.8.3.RD1

Source of Support: Nil, Conflict of Interest: None declared. 